\title{
Uniform Linear Arrays with Optimized Inter-Element Spacing for LOS Massive MIMO
}

\section{Citation for published version (APA):}

Farsaei, A., Amani, N., Maaskant, R., Gustavsson, U., Alvarado, A., \& Willems, F. M. J. (2021). Uniform Linear Arrays with Optimized Inter-Element Spacing for LOS Massive MIMO. IEEE Communications Letters, 25(2), 613-616. [9222215]. https://doi.org/10.1109/LCOMM.2020.3030681

DOI:

10.1109/LCOMM.2020.3030681

Document status and date:

Published: 01/02/2021

\section{Document Version:}

Accepted manuscript including changes made at the peer-review stage

\section{Please check the document version of this publication:}

- A submitted manuscript is the version of the article upon submission and before peer-review. There can be important differences between the submitted version and the official published version of record. People interested in the research are advised to contact the author for the final version of the publication, or visit the $\mathrm{DOI}$ to the publisher's website.

- The final author version and the galley proof are versions of the publication after peer review.

- The final published version features the final layout of the paper including the volume, issue and page numbers.

Link to publication

\section{General rights}

Copyright and moral rights for the publications made accessible in the public portal are retained by the authors and/or other copyright owners and it is a condition of accessing publications that users recognise and abide by the legal requirements associated with these rights.

- Users may download and print one copy of any publication from the public portal for the purpose of private study or research.

- You may not further distribute the material or use it for any profit-making activity or commercial gain

- You may freely distribute the URL identifying the publication in the public portal.

If the publication is distributed under the terms of Article 25fa of the Dutch Copyright Act, indicated by the "Taverne" license above, please follow below link for the End User Agreement:

www.tue.nl/taverne

Take down policy

If you believe that this document breaches copyright please contact us at:

openaccess@tue.nl

providing details and we will investigate your claim. 


\title{
Uniform Linear Arrays with Optimized Inter-Element Spacing for LOS Massive MIMO
}

\author{
A. Farsaei, N. Amani, R. Maaskant, Senior Member, IEEE, U. Gustavsson, \\ A. Alvarado, Senior Member, IEEE, F. M. J. Willems, Fellow, IEEE
}

\begin{abstract}
In this letter, a uniform linear array (ULA) is proposed for line-of-sight massive multiple-input-multiple-output (MIMO). It is assumed that the number of antennas is fixed. For a given ULA with an arbitrary inter-element spacing, the probability that the correlation among the channel vectors of two users being above a threshold value is derived. The inter-element spacing of the proposed ULA is the one for which the aforementioned probability is minimized. To show the effectiveness of the proposed ULA, simulation results for two scenarios are given for a 64 -antenna ULA that serves 6 single-antenna users. By using the proposed ULA instead of conventional half-wavelength ULA, 5 th percentile sum-rate for zero-forcing precoder is improved by 9.90 bits/channel use in first scenario without dropping, and by 1.43 bits/channel use in second scenario with dropping 1 user.
\end{abstract}

Index Terms-Line-of-sight, massive MIMO, uniform linear array, zero-forcing.

\section{INTRODUCTION}

Massive multiple-input-multiple-output (MIMO) is foreseen as a key enabling technology for fifth-generation wireless networks and beyond [1], [2]. It is shown in [3] that in line-of-sight (LOS) massive MIMO, there is a nonnegligible probability that the channel vectors of some users become highly correlated, which results in a non-favorable propagation environment. The high correlation leads to a reduction in the sum-rates of linear and nonlinear precoders [4, Fig. 5]. The reduction of the sum-rate due to the high correlation is considerable for LOS environments with max-min power control as reported in [5], [6] (max-min power control is used to provide uniformly good service for the users as reported in [3]). In addition, it is shown in [5, Fig. 2] that when there is only one pair of highly correlated users, the signal to noise ratio with max-min power control will drop significantly. To deal with highly correlated scenarios in LOS environments with max-min power control, [3], [5], [6] studied dropping algorithms. However, dropping users may not be desirable in the case of latency-sensitive communication.

To avoid dropping and alleviate a high inter-user correlation, one can increase the aperture size to improve the angular resolution of the base station (BS) antenna array. By increasing

A. Farsaei, A. Alvarado and F. M. J. Willems are with Eindhoven University of Technology, 5600 MB Eindhoven, The Netherlands (email: \{a.farsaee,a.alvarado,f.m.j.willems\}@tue.nl).

N. Amani and R. Maaskant are with Chalmers University of Technology, 41296 Gothenburg, Sweden (email: \{anavid, rob.maaskant\}@ chalmers.se).

U. Gustavsson is with Ericsson Research, Ericsson AB, 41756 Gothenburg, Sweden (email: ulf.gustavsson@ericsson.com).

This project has received funding from the European Union's Horizon 2020 research and innovation programme under the Marie Sklodowska-Curie grant agreement No 721732 . the aperture size, the minimum resolvability angular resolution of the array, which is defined by the well-known Rayleigh's criterion [7], is improved. Hence, by employing an interelement spacing $(\delta)$ larger than the conventional $\lambda / 2(\lambda$ is a wavelength) the angular resolution of an array with a fixed number of elements is enhanced [8, Sec. 7.2.4]. The major drawback of increasing $\delta$ in the uniform linear arrays (ULAs) is the appearance of grating lobes (beamforming ambiguities) [9], [10]. The grating lobes may cause a high correlation among the channel vectors of users with a large angular separation (not co-located users). To avoid grating lobes, in [11] a maximum allowable $\delta$, depending on the fieldof-view (FOV), is proposed, where the increase in the aperture size is minimal for wide FOVs. Increasing $\delta$ is reported beneficial in terms of spectral efficiency for a BS antenna array with a fixed number of antennas [12]. A small LOS spectral efficiency improvement is also reported in [10] by deploying ULAs with larger inter-element spacing. However, none of the above-mentioned studies approaches the problem analytically to compute the probability of correlated users in the absence or presence of grating lobes.

In this letter, a ULA for LOS environments is proposed assuming a fixed number of omnidirectional antennas at the BS. We derive the probability that the correlation among the channel vectors of two users being above a threshold for a ULA with an arbitrary inter-element spacing. The interelement spacing of the proposed ULA is the one for which the aforementioned probability is minimized. The proposed ULA is optimized for the case when there are only two users. For more users, we present simulation results for two different scenarios, to show the effectiveness of the proposed array compared to conventional half-wavelength ULA with a known linear precoder, i.e., zero-forcing (ZF).

\section{System Model}

We consider a BS equipped with a ULA of $M$ antennas ${ }^{1}$ located on the $x$-axis (see Fig. 1). Two users are assumed to be in the $x-y$ plane, where $R_{1}$ and $R_{2}$ are the distance from the users to the first element of the array, and $\phi_{1}$ and $\phi_{2}$ are the azimuth angles of the users. It is assumed that $\phi_{1}$ and $\phi_{2}$ are independent random variables that are uniformly distributed in a FoV of $\phi_{l} \in\left(\pi / 2-\phi_{o}, \pi / 2+\phi_{o}\right)$, where $\phi_{o} \in(0, \pi / 2)$. The channel between user $l(l \in\{1,2\})$ and antenna $m(m \in\{1,2, \ldots, M\})$ is modeled as [8, Sec. 7.2.2]:

$$
h_{l m}=\sqrt{\beta_{l}} e^{-\jmath k R_{l}} e^{+\jmath k(m-1) \delta \cos \left(\phi_{l}\right)},
$$

\footnotetext{
${ }^{1}$ Analysis of uniform planar array (3D beamforming) is left for future work.
} 


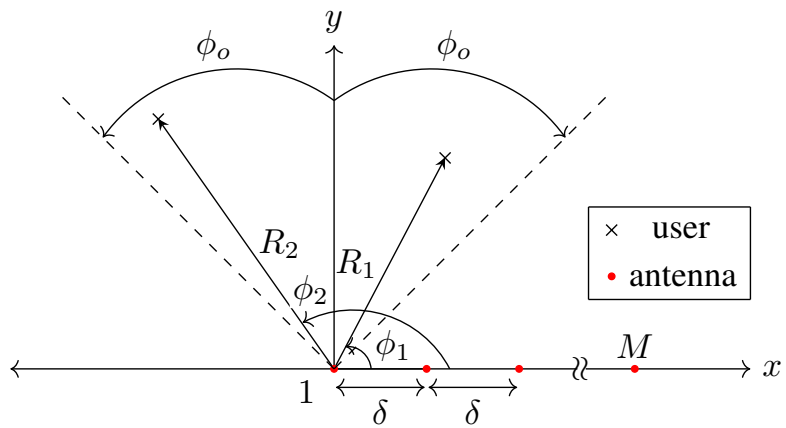

Fig. 1. ULA with $M$ elements on x-axis with inter-element spacing $\delta$. The distance between the first element of the array and the users are $R_{1}$ and $R_{2}$.

where $\beta_{l}$ is the large-scale fading for user $l, k$ is the wavenumber, and $\delta$ is the inter-element spacing of ULA. Typically, $\delta$ is assumed to be $\lambda / 2$. Using (1), the channel vector $\boldsymbol{h}_{l}=\left(h_{l 1}, h_{l 2}, \ldots, h_{l M}\right)^{T}$ is found. The spatial correlation between the channel vectors $\boldsymbol{h}_{1}$ and $\boldsymbol{h}_{2}$ is given by:

$$
\rho=\frac{\boldsymbol{h}_{2}^{H} \boldsymbol{h}_{1}}{\left\|\boldsymbol{h}_{1}\right\|\left\|\boldsymbol{h}_{2}\right\|} .
$$

We use the term spatial correlation for (2) (following the literature) throughout the paper, although, (2) is the innerproduct of normalized $\boldsymbol{h}_{1}$ and $\boldsymbol{h}_{2}$ for a given coherence interval. By replacing the elements of the channel vectors $\boldsymbol{h}_{1}$ and $\boldsymbol{h}_{2}$ using (1), in (2), $|\rho|$ is found by:

$$
|\rho|=\frac{1}{M}\left|\frac{\sin \left(M k \frac{\delta}{2} \psi\right)}{\sin \left(k \frac{\delta}{2} \psi\right)}\right|,
$$

where $\psi=\cos \left(\phi_{1}\right)-\cos \left(\phi_{2}\right)$. Using (3), $|\rho|$ is expressed as a function of $\psi$ with the inter-element spacing of $\delta$ as follows:

$$
|\rho|=f_{\delta}(\psi), \quad \psi \in\left(2 \cos \left(\frac{\pi}{2}+\phi_{o}\right), 2 \cos \left(\frac{\pi}{2}-\phi_{o}\right)\right) .
$$

Note $f_{\delta}(\psi)$ is periodic with period $T=\lambda / \delta$ [8, Sec. 7.2.4].

For a given realization of a channel of two users, assume that the angular separation of the users is $\psi=\Delta$. One can find the inter-element spacing $\delta_{1}$ such that the users become orthogonal, i.e., $|\rho|=f_{\delta_{1}}(\Delta)=0$. Suppose the users move and the angular separation of the users becomes $\Delta^{\prime} \neq \Delta$. In this case, another inter-element spacing $\delta_{2} \neq \delta_{1}$ has to be used to make the users orthogonal. However, changing the interelement spacing for each realization of users is not practical. Therefore, a probabilistic approach is required to find the best inter-element spacing $\delta^{\star}$ for which a small $|\rho|$ is achieved with a high probability. In other words, the best inter-element spacing is the one that has the minimum probability that $|\rho|$ becomes larger than a given threshold $\rho_{o}$. We use the following definition for the rest of the paper.

Definition 1. The probability that a pair of users with the spatial correlation of $\rho$ become correlated with a given $\rho_{o}$, is denoted by $p$, and defined as:

$$
p \triangleq \operatorname{Pr}\left\{|\rho|>\rho_{o}\right\} .
$$

Appropriate probability analysis is required to find the interelement spacing for the case of two users for which $p$ is minimized, which is given in the sequel.

\section{PRobability ANALYSis}

In this section, we find $p$ for ULAs with $\delta=\lambda / 2$ and then for ULAs with $\delta>\lambda / 2$ when there are only two users.

\section{A. ULAs with $\delta=\lambda / 2$}

In Fig. 2, $|\rho|=f_{\lambda / 2}(\psi)$ is shown for a ULA of $M=$ 10 antennas. The shaded areas show when user 1 and user 2 become correlated with a given $\rho_{o}=0.64\left(\rho_{o}=0.64\right.$ is the 3 -dB point $\left[9\right.$, Sec. 6.3]) or equivalently a given $\psi_{o}$. If $\psi_{o}$ is chosen as in Fig. 2, we can derive $p$ as follows using the periodicity of $f_{\lambda / 2}(\psi)(T=2)$ :

$$
p=\operatorname{Pr}\left\{|\psi|<\psi_{o}\right\}+\operatorname{Pr}\left\{2-\psi_{o}<|\psi|<2\right\}=\alpha_{0}+\alpha_{1},
$$

where the corresponding area for $\alpha_{0}$ and $\alpha_{1}$ are shown in Fig. 3 by the blue and yellow shaded area, respectively. We find $\alpha_{0}$ as follows:

$$
\begin{aligned}
\alpha_{0} & =\operatorname{Pr}\left\{|\psi|<\psi_{o}\right\}=2 \operatorname{Pr}\left\{0 \leq \psi<\psi_{o}\right\} \\
& =2 \operatorname{Pr}\left\{0 \leq \cos \left(\phi_{1}\right)-\cos \left(\phi_{2}\right)<\psi_{o}\right\} \\
& =2 \operatorname{Pr}\left\{\cos \left(\phi_{2}\right) \leq \cos \left(\phi_{1}\right)<\cos \left(\phi_{2}\right)+\psi_{o}\right\} \\
& =2 \operatorname{Pr}\left\{\cos ^{-1}\left(\cos \left(\phi_{2}\right)+\psi_{o}\right)<\phi_{1} \leq \phi_{2}\right\} .
\end{aligned}
$$

By using the same approach, $\alpha_{1}$ is found by:

$$
\begin{aligned}
\alpha_{1} & =\operatorname{Pr}\left\{2-\psi_{o}<|\psi|<2\right\} \\
& =2 \operatorname{Pr}\left\{2-\psi_{o}<\psi<2\right\} \\
& =2 \operatorname{Pr}\left\{2-\psi_{o}<\cos \left(\phi_{1}\right)-\cos \left(\phi_{2}\right)<2\right\} \\
& =2 \operatorname{Pr}\left\{\cos \left(\phi_{2}\right)+2-\psi_{o}<\cos \left(\phi_{1}\right)<\cos \left(\phi_{2}\right)+2\right\} .
\end{aligned}
$$

Note $\cos \left(\phi_{2}\right)+2>1$ for all $\phi_{2}$ in the FoV. Therefore:

$$
\begin{aligned}
\alpha_{1} & =2 \operatorname{Pr}\left\{\cos \left(\phi_{2}\right)+2-\psi_{o}<\cos \left(\phi_{1}\right)<1\right\} \\
& =2 \operatorname{Pr}\left\{\frac{\pi}{2}-\phi_{o}<\phi_{1}<\cos ^{-1}\left(\cos \left(\phi_{2}\right)+2-\psi_{o}\right)\right\} .
\end{aligned}
$$

Recall that $\phi_{l}$ with $l=1,2$ are uniformly distributed in the FoV. Consequently, given (7)-(9), $\alpha_{0}$ and $\alpha_{1}$ are found by evaluating the following integrals:

$$
\begin{aligned}
& \alpha_{0}=2 \int_{\frac{\pi}{2}-\phi_{o}}^{\frac{\pi}{2}+\phi_{o}} \frac{1}{2 \phi_{o}} \int_{\cos ^{-1}\left(\cos \left(\phi_{2}\right)+\psi_{o}\right)}^{\phi_{2}} \frac{1}{2 \phi_{o}} d \phi_{1} d \phi_{2}, \\
& \alpha_{1}=2 \int_{\frac{\pi}{2}-\phi_{o}}^{\frac{\pi}{2}+\phi_{o}} \frac{1}{2 \phi_{o}} \int_{\frac{\pi}{2}-\phi_{o}}^{\cos ^{-1}\left(\cos \left(\phi_{2}\right)+2-\psi_{o}\right)} \frac{1}{2 \phi_{o}} d \phi_{1} d \phi_{2} .
\end{aligned}
$$

Whenever $\rho_{o}$ is higher than the black squares (first side-lobes at $\rho_{o}=2 /(3 \pi)[9$, Sec. 6.3]) shown in Fig. 2, $p$ can be written as a sum of $\alpha_{0}$ and $\alpha_{1}$ in (6), where $\alpha_{0}$ and $\alpha_{1}$ are found by (10) and (11), respectively.

\section{B. ULAs with $\delta>\lambda / 2$}

In this section, we first find $p$ for a ULA with $\delta=\lambda$. Then, we give an expression for ULAs with any $\delta>\lambda / 2$. In Fig. 3, $|\rho|=f_{\lambda}(\psi)$ is shown for a ULA of $M=10$ antennas. The shaded areas show when user 1 and user 2 become correlated with a given $\rho_{o}$. The probability $p$ is found by:

$$
\begin{aligned}
& p=\operatorname{Pr}\left\{|\rho|>\rho_{o}\right\} \\
& \quad=\operatorname{Pr}\left\{|\psi|<\psi_{o}\right\}+\operatorname{Pr}\left\{1-\psi_{o}<|\psi|<1+\psi_{o}\right\}+ \\
& \operatorname{Pr}\left\{2-\psi_{o}<|\psi|<2\right\}=\alpha_{0}+\alpha_{1}+\alpha_{2},
\end{aligned}
$$




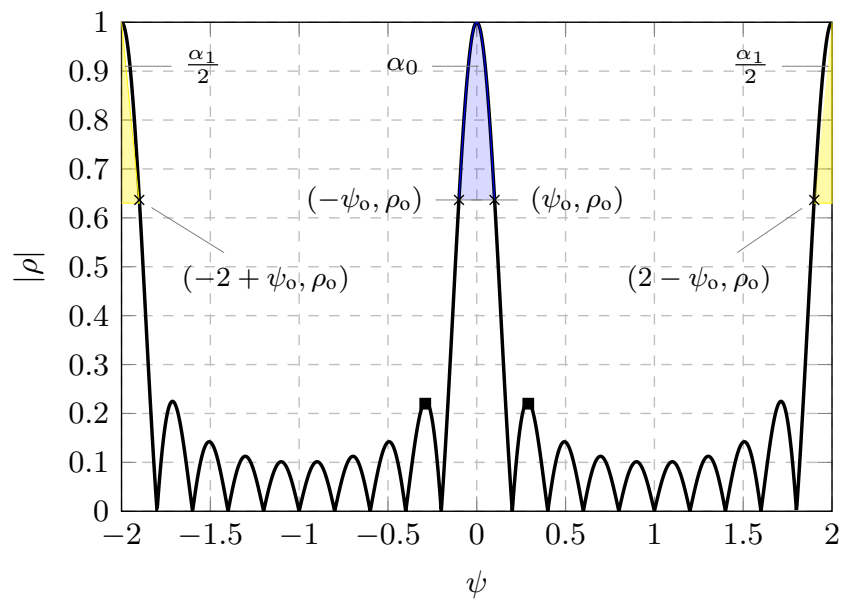

Fig. 2. The function $|\rho|=f_{\lambda / 2}(\psi)$ for a ULA with $M=10$ and $\psi_{o}=0.1$ $\left(\rho_{o}=0.64\right)$ for a FoV of $(0, \pi)$.

where the corresponding area for $\alpha_{0}, \alpha_{1}$, and $\alpha_{2}$ are shown in Fig. 3 by blue, red, and yellow shaded area, respectively. Similar to case of $\delta=\lambda / 2$, we find integrals for $\alpha_{0}, \alpha_{1}$, and $\alpha_{2}$. For a ULA with $\delta>\lambda / 2, p$ for a given $\rho_{o}$ is found by:

$$
\begin{aligned}
& p=\operatorname{Pr}\left\{|\rho|>\rho_{o}\right\}=\operatorname{Pr}\left\{|\psi|<\psi_{o}\right\}+ \\
& \sum_{i=1}^{n} \operatorname{Pr}\left\{i T-\psi_{o}<|\psi|<i T+\psi_{o}\right\}=\alpha_{0}+\sum_{i=1}^{n} \alpha_{i},
\end{aligned}
$$

where $n$ is the number of areas where $\psi>0$ and $\rho>\rho_{o}$ excluding the area corresponds to $\alpha_{0}$. For instance, $n=2$ for $\delta=\lambda$. We numerically evaluate integrals to find $\alpha_{0}$ and $\alpha_{i}$ to find $p$, same as the analysis done for $\lambda / 2$.

In Fig. 4, $p$ is shown as a function of $\delta / \lambda$ for $M=10,20,64$ for $\delta \leq 2.5 \lambda^{2}, \rho_{o}=0.64$ for a FoV of $(0, \pi)$. For each $M, \delta^{\star}$ shows the inter-element spacing with minimum $p$, and there are three local minima as shown by colored circles $\delta_{n_{1}}, \delta_{n_{2}}$, and $\delta_{n_{3}}$. By increasing $\delta, p$ is continuously increasing and then decreasing with decaying behavior. There are two reasons for explaining this behavior. First, by increasing $\delta$, the angular resolution of the array is improved (compare the shaded blue area in Fig. 2 and Fig. 3), which decreases $p$. Second, by increasing $\delta$, the grating lobes (the peaks correspond to $\alpha_{i}, i \neq$ 0 , see Fig. 3) gradually appear in the FoV, which increases $p$. Due to the first reason, $p$ should decrease, and due to the second reason, $p$ should increase. As can be seen, we see a more decrease in $p$, which shows that increasing the angular resolvability has a stronger effect on $p$ than the grating lobes. Moreover, the effect of increasing the angular resolvability and the appearance of grating lobes is decaying as $\delta$ approaches $2.5 \lambda$. We further observe that in all the scenarios, $p$ curves approach $p=1 / M$ (horizontal dash-dotted lines).

We approximate $\delta_{n_{i}}$ by the inter-element spacing for which the shaded area associated with $(i+1)$ th grating lobe appeared in function $f_{\delta}(\psi)$ (e.g., in Fig. 3, the yellow shaded area is associated with the 2 nd grating lobe). For instance, $\delta_{n_{1}}$ is approximated by the inter-element spacing for which the yellow area starts to appear in Fig. 3. For a given $\psi_{o}$, we approximate $\delta_{n_{i}}$ by solving $\psi_{o}-(i+1) \lambda / \delta=-2$ for $\delta$, which leads to $\delta_{n_{i}} \approx \lambda(2 M(i+1)-1) / 4 M$ for $\psi_{o}$ of the scenario

\footnotetext{
${ }^{2}$ The choice of $2.5 \lambda$ is arbitrary to limit the maximum aperture size.
}

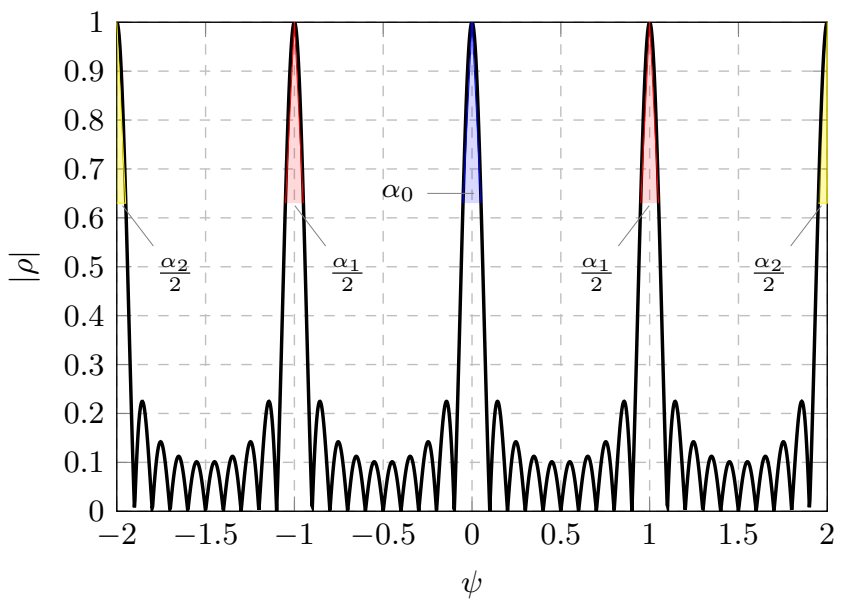

Fig. 3. The function $|\rho|=f_{\lambda}(\psi)$ for $M=10, \rho_{o}=0.64, \psi_{o}=0.05$, and for a FoV of $(0, \pi)$. The blue, red, and yellow shaded area are associated with $\alpha_{0}, \alpha_{1}$, and $\alpha_{2}$, respectively. Note that $T=1$.

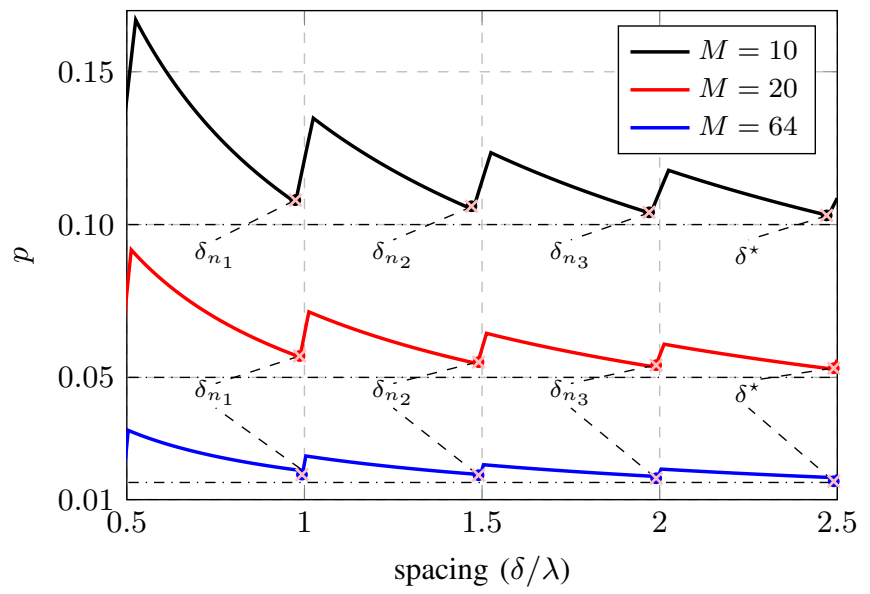

Fig. 4. The probability $p\left(\rho_{o}=0.64\right)$ as a function of $\delta / \lambda$ for $M=$ $10,20,64$ for a FoV of $(0, \pi)$ for $\delta \in[0.5 \lambda, 2.5 \lambda]$. For each $M$, the minimum $p$ occurs at $\delta^{\star}$. The local minima are $\delta_{n_{1}}, \delta_{n_{2}}$ and $\delta_{n_{3}}$.

in Fig. 4. The results in Fig. 4 shows that the approximations of $\delta_{n_{i}}$ (pink cross) match with the numerical values of $\delta_{n_{i}}$ (colored circle). For a given $M$, we propose to use $\delta^{\star}$, which is the inter-element spacing with the minimum $p$. To reduce the aperture size, one can use $\delta_{n_{i}}, i=1,2,3$ instead of $\delta^{\star}$. We assume a narrow-band communication system in this letter. However, the results in Fig. 4 can be used for multi sub-carriers systems. By choosing an appropriate spacing for the center sub-carrier, one can make $p$ smaller than a threshold for all the sub-carriers. The performance of using $\delta_{n_{1}}$ and $\delta^{\star}$ for more number of users is compared in the next Section. Regarding scenarios where there are paths other than LOS path some insights can be found in [13].

\section{Simulation Results}

In this section, the performance of ULAs with $\delta^{\star}$ and $\delta_{n_{1}}$ (see Fig. 4) are compared with half-wavelength ULA for FoV of $(0, \pi)$ in LOS massive MIMO with max-min power control. To study the worst-case scenarios, the users are assumed to be at the cell-edge (no shadowing), which is assumed to be at the far-field of the array. We compare the arrays qualitatively and quantitatively as follows. First, qualitatively, for a given $\rho_{o}$, we 


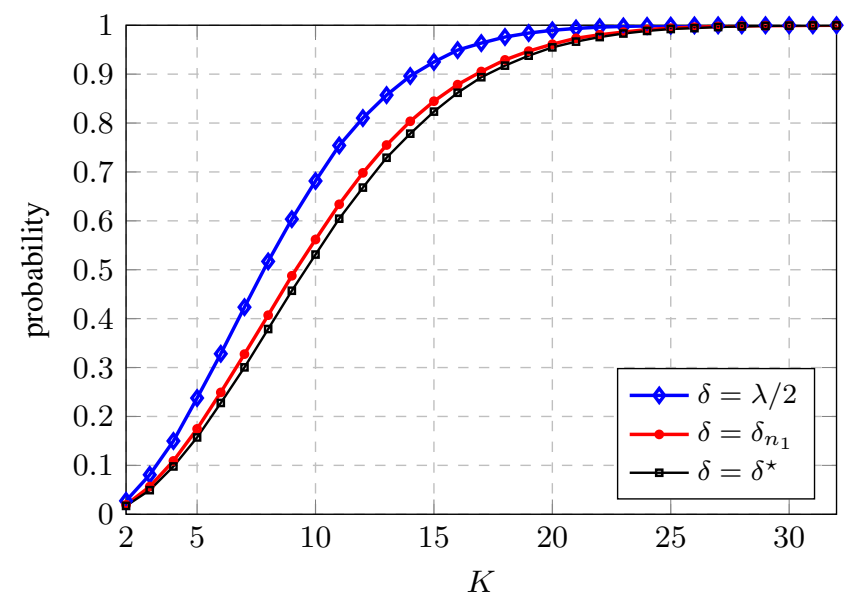

Fig. 5. The probability that there is at least one pair of correlated users for $\delta=\lambda / 2, \delta_{n_{1}}, \delta^{\star}$ when $M=64, \rho_{o}=0.64$ for a FoV of $(0, \pi)$.

compare the probability that at least there is one correlated pair of users as a function of the number of users for the three arrays. Second, quantitatively, we compare cumulative distribution function (CDF) of $\mathrm{ZF}$ sum-rates of the arrays.

In Fig. 5, for a ULA with $M=64$ antennas, the probability that there is at least one pair of correlated users $\left(\rho_{o}=0.64\right)$ is shown as a function of the number of users $K$ for $\delta=\lambda / 2$ (blue), $\delta_{n_{1}}$ (red), and $\delta^{\star}$ (black). For a given number of users, the ULA with $\delta^{\star}$ has a smaller probability compared to $\lambda / 2$, which means it has a better ability to decorrelate the channel vectors of the users. By using $\delta_{n_{1}}$ instead of $\delta^{\star}$, we can reduce the aperture size, while the probability that there is at least one pair of correlated users is not that higher than that of $\delta^{\star}$.

In Fig. 6, the CDF of ZF sum-rate is shown for the arrays with $K=6$ and $M=64$ in two different scenarios, where $100 \mathrm{~K}$ realizations of users' locations are drawn for each scenario. In the first scenario, no user is dropped (No Dropping), while in the second scenario one user is dropped (Drop 1 user) based on the dropping algorithm of [3]. The transmit power at the BS is fixed and is the same in both scenarios such that in the favorable propagation (FP) [14] (when the users are mutually orthogonal), a sum-rate of 36 bits/channel use is achieved in the first scenario, and a sumrate of 31.3 bits/channel use is achieved in the second scenario (see the vertical dashed lines in Fig. 6). When no user is dropped, by employing the proposed array (black), the 5th percentile sum-rate is improved significantly $(9.90 \mathrm{bits} / \mathrm{channel}$ use) compared to that of the ULA with $\delta=\lambda / 2$ (blue). This improvement becomes 1.43 bits/channel use when 1 user is dropped. By dropping 1 user, the 5th percentile ZF sum-rate of all the arrays is improved significantly, which shows it is necessary to drop 1 user. To reduce the aperture size, the array with $\delta_{n_{1}}$ (red) can be used instead of $\delta^{\star}$ with a loss in performance, i.e., 3.30 bits/channel use loss in No Dropping scenario and 0.09 bits/channel use in Drop 1 user scenario.

\section{CONCLUSIONS}

In this letter, we use probability analysis to find an improved uniform linear array for LOS massive MIMO. For the case of two users, the proposed ULA has the minimum probability that the correlation of the users being above a given threshold. For

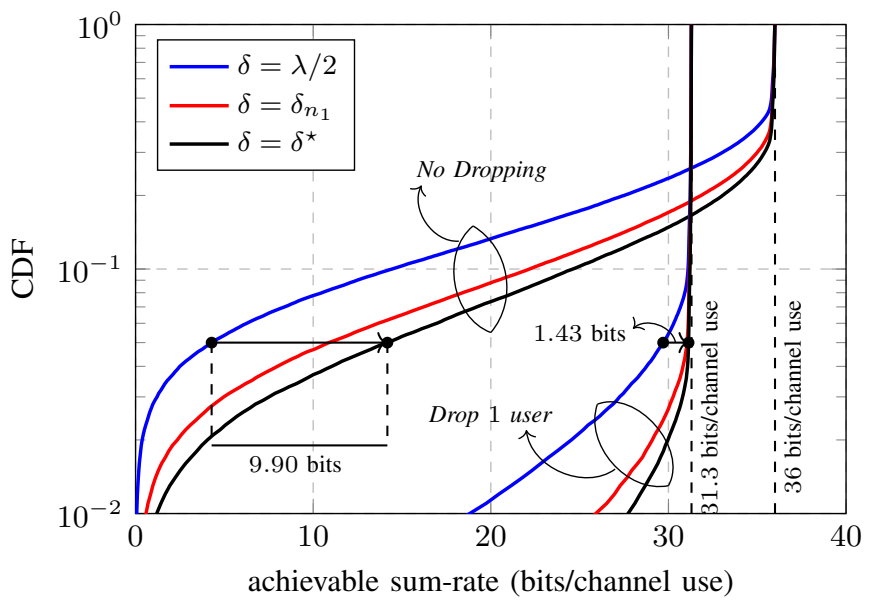

Fig. 6. The CDF plots of ZF sum-rate for $\delta=\lambda / 2, \delta_{n_{1}}, \delta^{\star}$ for two different scenarios, i.e., No dropping and Drop 1 user (based on the algorithm of [3]). The horizontal arrow shows the 5th percentile improvement of the sum-rate by using $\delta^{\star}$ instead of $\delta=\lambda / 2$. The vertical dashed lines show the ZF sum-rate in FP for the two scenarios.

more users, we present the simulation results for a known linear precoder, i.e., $\mathrm{ZF}$ to show the effectiveness of the proposed ULA compared to half-wavelength ULA.

\section{REFERENCES}

[1] T. L. Marzetta, "Noncooperative cellular wireless with unlimited numbers of base station antennas," IEEE transactions on wireless communications, vol. 9, no. 11, pp. 3590-3600, 2010.

[2] E. G. Larsson, O. Edfors, F. Tufvesson, and T. L. Marzetta, "Massive MIMO for next generation wireless systems," IEEE Communications Magazine, vol. 52, no. 2, pp. 186-195, Feb. 2014.

[3] H. Yang and T. L. Marzetta, "Massive MIMO with max-min power control in line-of-sight propagation environment," IEEE Transactions on Communications, vol. 65, no. 11, pp. 4685-4693, Nov. 2017.

[4] X. Gao, O. Edfors, F. Rusek, and F. Tufvesson, "Linear pre-coding performance in measured very-large MIMO channels," in 2011 IEEE Vehicular Technology Conference (VTC Fall), Sep. 2011, pp. 1-5.

[5] A. Farsaei, A. Alvarado, F. M. J. Willems, and U. Gustavsson, "An improved dropping algorithm for line-of-sight massive MIMO with maxmin power control," IEEE Communications Letters, vol. 23, no. 6, pp. 1109-1112, Jun. 2019.

[6] A. Farsaei, A. Alvarado, F. M. J. Willems, and U. Gustavsson, "An improved dropping algorithm for line-of-sight massive MIMO with Tomlinson-Harashima Precoding," IEEE Communications Letters, vol. 23, no. 11, pp. 2099-2103, Nov. 2019.

[7] E. Hecht, Optics, ser. Pearson education. Addison-Wesley, 2002.

[8] D. Tse and P. Viswanath, Fundamentals of Wireless Communication. New York, NY, USA: Cambridge University Press, May 2005.

[9] C. A. Balanis, Antenna theory: analysis and design. John wiley \& sons, 2016

[10] S. Pratschner, E. Zöchmann, H. Groll, S. Caban, S. Schwarz, and M. Rupp, "Does a large array aperture pay off in line-of-sight massive MIMO?" in 2019 IEEE 20th International Workshop on Signal Processing Advances in Wireless Communications, Jul. 2019, pp. 1-5.

[11] N. Amani, A. A. Glazunov, M. V. Ivashina, and R. Maaskant, "Perantenna power distribution of a zero-forcing beamformed ULA in pure LOS MU-MIMO," IEEE Communications Letters, vol. 22, no. 12, pp. 2515-2518, Dec. 2018.

[12] D. Pinchera, M. D. Migliore, F. Schettino, and G. Panariello, "Antenna arrays for line-of-sight massive MIMO: Half wavelength is not enough," Electronics, vol. 6, no. 3, p. 57, 2017.

[13] N. Amani, A. Farsaei, U. Gustavsson, T. Eriksson, F. M. J. Willems, M. V. Ivashina, and R. Maaskant, "Array configuration effect on the spatial correlation of MU-MIMO channels in NLoS environments," in 2020 14th European Conference on Antennas and Propagation (EuCAP), 2020, pp. 1-4.

[14] H. Q. Ngo, E. G. Larsson, and T. L. Marzetta, "Aspects of favorable propagation in massive MIMO," in 2014 22nd European Signal Processing Conference (EUSIPCO), Sep. 2014, pp. 76-80. 